\title{
Experimental observation of steady inertial wave turbulence in deep rotating flows
}

\author{
Ehud Yarom and Eran Sharon ${ }^{\star}$
}

The theoretical framework that should be used for describing rotating turbulence ${ }^{1-3}$ is the subject of an active debate. It was shown experimentally ${ }^{4,5}$ and numerically ${ }^{6,7}$ that the formalism of 2D turbulence is useful in the description of many aspects of rotating turbulence. On the other hand, theoretical and numerical work suggests that the formalism of wave turbulence ${ }^{8-10}$ should provide a reliable description of the entire 3D flow field ${ }^{11-15}$. The waves that are suggested as the basis for this turbulence are Coriolis-force-driven inertial waves $^{1}$. Here we present experimental results that suggest the existence of inertial wave turbulence in deep steady rotating turbulence. Our measurements show energy transfer from the injection scale to larger scales, although the energy spectra are concentrated along the dispersion relation of inertial waves. The turbulent fields are, therefore, well described as ensembles of 3D interacting inertial waves.

Rotating flows are described by the rotating Navier-Stokes equation ${ }^{1}$. For incompressible fluids this equation is characterized by two dimensionless numbers: the Reynolds number, $\operatorname{Re}=U L / v$, and the Rossby number, Ro $=U / 2 \Omega L$ ( $U$ and $L$ are the typical velocity and length in the flow, $v$ the kinematic viscosity and $\Omega=|\boldsymbol{\Omega}|$ the rotation rate of the system). Rotating turbulence is obtained for $\operatorname{Re} \gg 1$ and Ro $<1$. Such flows, which are common in various geophysical and planetary systems, have been studied extensively ${ }^{16}$. It was found that the horizontal component of the velocity field in such flows shares many similarities with $2 \mathrm{D}$ turbulence. In particular, the cascade of energy to large scales ${ }^{5,7,17}$, the formation of coherent structures ${ }^{18}$ and the self-similarity of the flow field ${ }^{4}$ appear in both types of flows. However, the $2 \mathrm{D}$ description cannot describe the entire $3 \mathrm{D}$ rotating turbulent field. In addition, it is not obtained rigorously from the rotating Navier-Stokes equation.

For small enough Ro (fast rotation), the linearized (rotating) Navier-Stokes equation supports the propagation of waves, known as inertial waves ${ }^{1}$. Each component of the velocity field, $\mathbf{u}=\left(u_{x}, u_{y}, u_{z}\right)$, varies in space and time via the propagation of plane waves of the form $u_{x, y, z} \propto \mathrm{e}^{\mathrm{i}(\mathbf{k} \cdot \mathbf{r}+\omega t)}$, where $\omega$ and $\mathbf{k}$ are the wave frequency and wavevector, respectively. These waves obey a unique dispersion relation:

$$
\omega= \pm \frac{2(\mathbf{k} \cdot \boldsymbol{\Omega})}{|\mathbf{k}|}= \pm 2 \Omega \cos (\theta)
$$

where $\theta$ is the angle between $\mathbf{k}$ and $\boldsymbol{\Omega}$.

This dispersion relation has some unusual properties: The group and phase velocities are perpendicular to each other; there is a cutoff frequency of $2 \Omega$, above which inertial waves do not exist; the wave frequency, $\omega$, defines only the orientation of the wavevector, $\hat{\mathbf{k}}$, not its magnitude.

It was suggested ${ }^{12,14,15}$ that weak steady rotating turbulence (moderate Re and low Ro) should be well described as the wave

turbulence of 3D interacting inertial waves. In wave turbulence, the energy is contained and transferred by interacting waves. As such, a continuous energy spectrum is built along the dispersion relation curve of the linear waves of the system. The theory of wave turbulence provided explicit predictions for the energy spectra and energy transfer in various systems (see refs 8-10,19 and references therein). Some of these predictions were experimentally confirmed in systems such as surface waves ${ }^{19-21}$ and elastic bending waves ${ }^{22-24}$. Being a rigorous $3 \mathrm{D}$ approximation, inertial wave turbulence, if valid, would provide an excellent framework for the study of rotating turbulence. However, rotating inertial wave turbulence has never been measured experimentally.

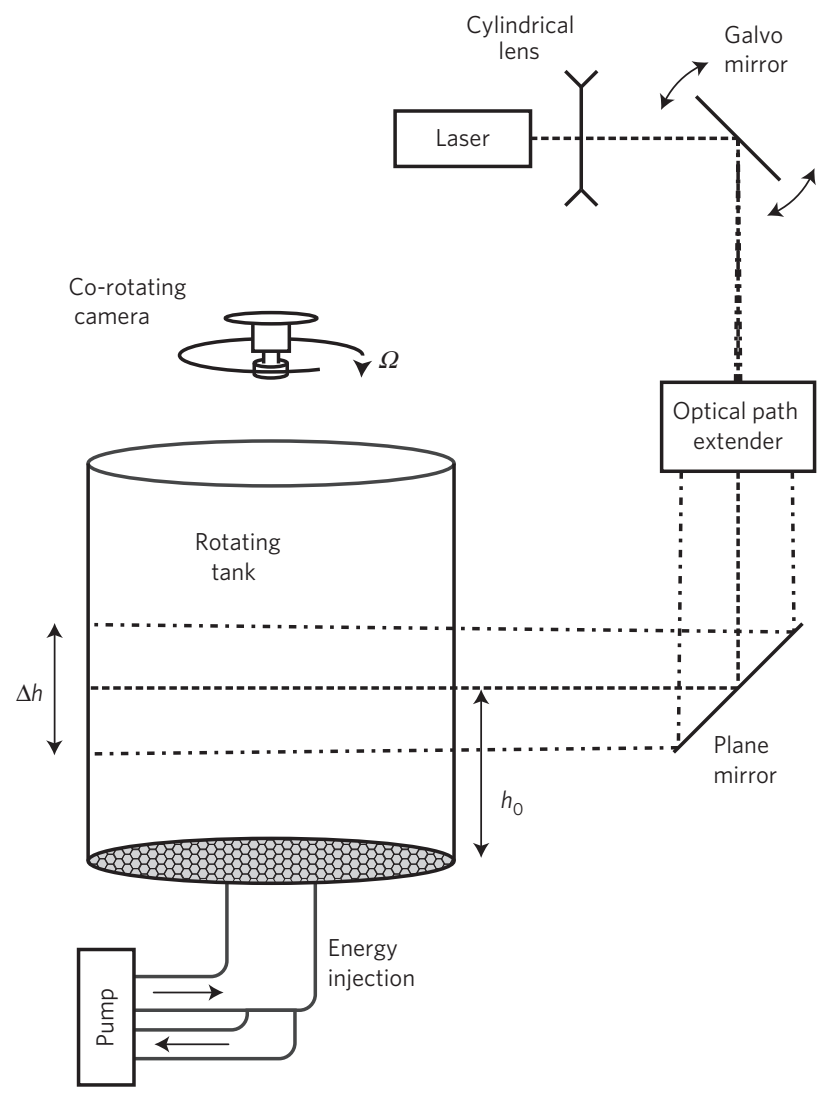

Figure 1 | Experimental set-up. A laser sheet illuminates a rotating Plexiglas cylinder filled with water and seeded with tracer particles. Using a galvo mirror, the sheet is repeatedly swept vertically through 30 horizontal planes, in the range $\Delta h=25.6 \mathrm{~cm}$ around height $h_{0}$. A co-rotating camera ( 750 frames per second) images the light scattered from the tracer particles. 
a

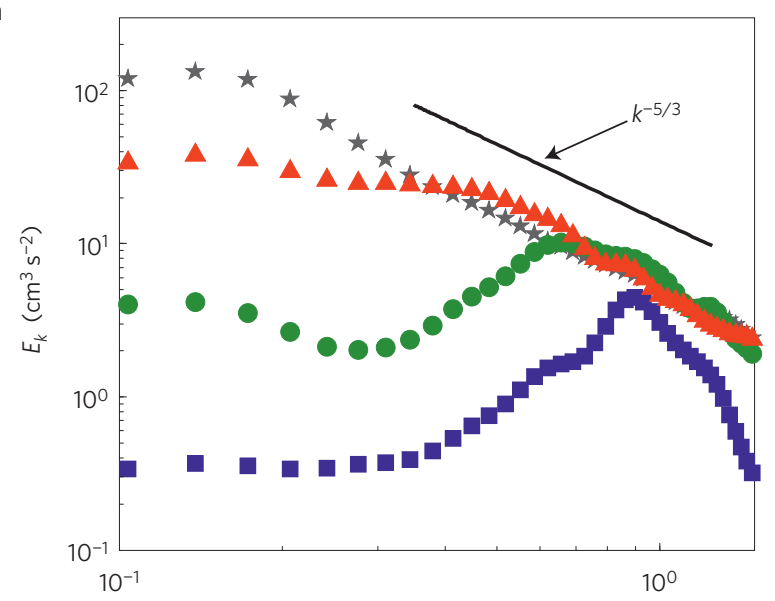

$|k|\left(\mathrm{rad} \mathrm{cm}^{-1}\right)$ b

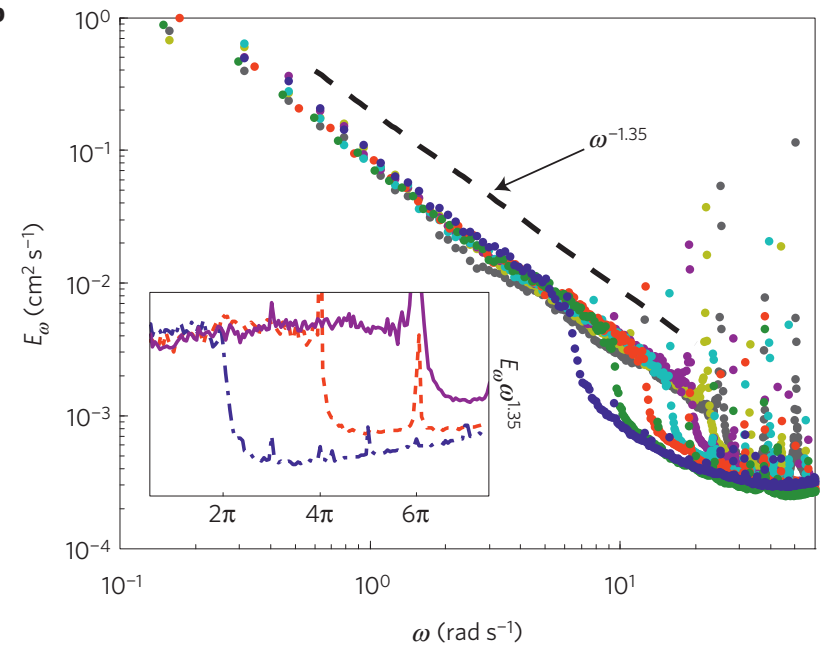

c

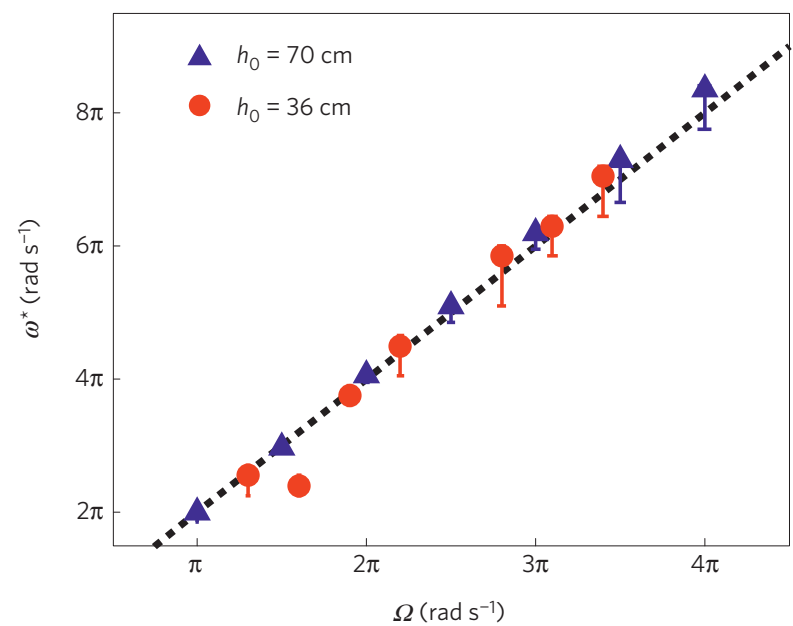

Figure 2 | 1D energy spectra in time or space for 2D measurements. a, Evolution of the energy spectrum $\left(\Omega=3 \pi\right.$ rad s$^{-1}$, measurement area $A=52 \times 52 \mathrm{~cm}^{2}$ ) at different times: $t(\mathrm{~s})=5.0$ (blue filled squares); 10.5 (green filled circles); 34.7 (red filled triangles); steady state (grey filled stars). At short times the energy injection scale is visible as a high peak at $k=0.87 \mathrm{rad} \mathrm{cm}^{-1}$ (Supplementary Information 2, Supplementary Figs 3 and $4 a$ ). At longer times the energy gradually occupies larger scales (Supplementary Fig. 4b). The final steady state has a power law of $k^{-5 / 3}$ (solid line). b, Averaged energy density spectrum at different rotation rates $\left(h_{0}=70 \mathrm{~cm}\right): \Omega\left(\mathrm{rad} \mathrm{s}^{-1}\right)=1 \pi$ (blue filled circles); $1.5 \pi$ (green filled circles); $2 \pi$ (red filled circles); $2.5 \pi$ (cyan blue filled circles); $3 \pi$ (purple filled circles); $3.5 \pi$ (chartreuse green filled circles); $4 \pi$ (grey filled circles). At low frequencies all measurements have the same energy spectra, with a power law of $\omega^{-1.35 \pm 0.05}$. At a cutoff frequency $\omega^{\star}(\Omega)$ there is a sharp decrease in energy. The inset shows the compensated energy spectrum $E_{\omega} \omega^{1.35}$ for three experiments: $\Omega\left(\right.$ rad s $\left.^{-1}\right)=1 \pi$ (blue dash-dotted), $2 \pi$ (red dashed) and $3 \pi$ (purple solid). The sharp decrease of energy can clearly be seen. The spikes at $\omega>\Omega$ are measurement noise and correspond to the rotation rate $(\Omega)$ and its harmonics. c, The cutoff frequency $\left(\omega^{\star}\right)$ as a function of rotation rate $(\Omega)$ for two different heights. $\omega^{\star}$ is defined as the frequency above which the energy spectrum no longer coincides with the power-law slope. Owing to the above-mentioned noise, $\omega^{\star}$ can be determined with error bars as follows: the error value for $\omega<\omega^{\star}$ is defined as the width of the spike at $\omega=2 \Omega$, whereas the error value for $\omega>\omega^{\star}$ is defined as the resolution of the measurement. The black dotted line indicates $\omega^{\star}=2 \Omega$.

Experiments did show that inertial waves are emitted from coherent, weak sources in rotating fluids ${ }^{1,25}$. In addition, components of inertial waves were measured during the early stages of turbulence build-up ${ }^{26,27}$, or the late stages of its decay ${ }^{28}$. However, the unusual dispersion relation (equation (1)) makes it difficult to directly measure these waves within turbulence: the wavevector $\mathbf{k}$ is related to the wave frequency $\omega$ only via its orientation. Therefore, verifying the dominance of these linear waves requires $3 \mathrm{D}$ information about the velocity field.

We describe an experimental system that clearly resolves the variation of the horizontal velocity field, $\mathbf{u}_{\perp}(x, y, z, t)=$ $\left(u_{x}(x, y, z, t), u_{y}(x, y, z, t)\right)$, in three dimensions together with its temporal variation. The measurements cover three decades in time and one and a half decades in (3D) length scale, allowing transformation into 3D spatial Fourier modes plus temporal Fourier modes. This 4D Fourier decomposition allows direct measurements of inertial waves in steady turbulence.

The experimental set-up is composed of a rotating Plexiglas cylinder, $90 \mathrm{~cm}$ in height and $80 \mathrm{~cm}$ in diameter (see Fig. 1), which rotates at angular velocities between $\pi$ and $4 \pi \operatorname{rads}^{-1}$ (with $\boldsymbol{\Omega}$ along the $z$ direction). The cylinder is filled with water seeded with $50 \mu \mathrm{m}$ white polyamide particle tracers, and closed with a clear flat lid. Energy is injected by circulating the water through 248 soft outlet tubes (silicon, $0.8 \mathrm{~mm}$ inner diameter, $12.5 \mathrm{~cm}$ length) and 73 inlets (6 $\mathrm{mm}$ diameter), which are arranged in overlapping hexagonal grids $(4.1 \mathrm{~cm}$ and $8.2 \mathrm{~cm}$ spacing, respectively) at the bottom of the tank. This device injects kinetic energy at a scale close to $7 \mathrm{~cm}$ (Supplementary Information 2 and Supplementary Fig. 3). In this paper all experiments are with moderate Reynolds number, $\operatorname{Re} \sim 1,500-5,000$, and small Rossby number, Ro $\sim 0.006-0.02$. The 

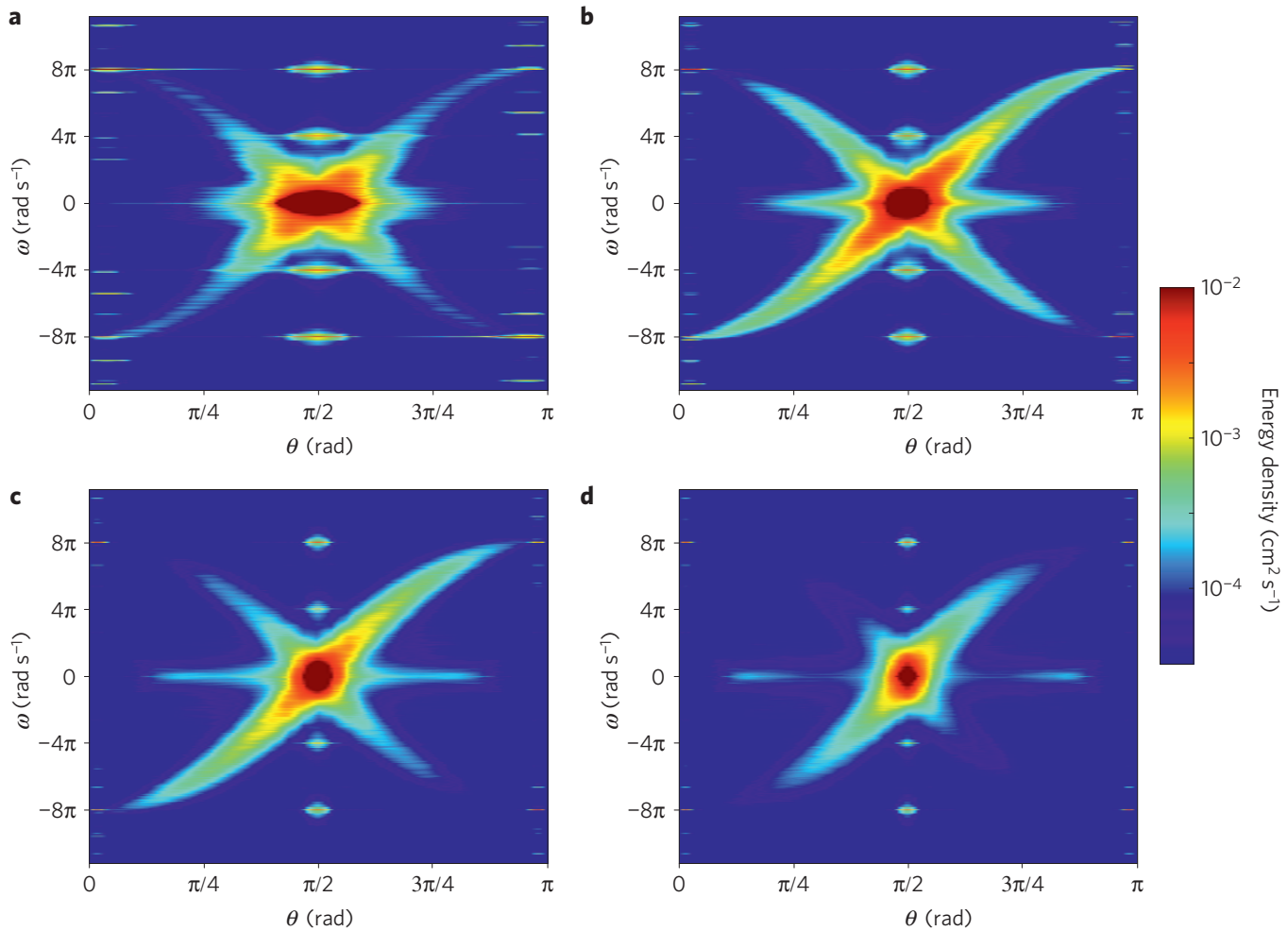

Figure 3 | Direct measurements of the inertial wave spectrum. The energy spectrum is localized along curves that correspond to the dispersion relation (equation (1)) of inertial waves. Integration across different wavenumber ranges (0.78-1.39 (a); 1.42-2.03 (b); 2.06-2.64 (c); $2.67-3.28$ (d) rad cm ${ }^{-1}$ ) shows that the shape of the curve is independent of $|k|$. In the high wavenumber range $(\mathbf{c}, \mathbf{d})$, the upward propagating waves carry more energy than the downward propagating waves. The data were taken at $h_{0}=68.5 \mathrm{~cm}$, with $\Delta h=25.9 \mathrm{~cm}$ and $\Omega=4 \pi \mathrm{rad} \mathrm{s}^{-1}$. The spikes at $\theta=\pi / 2$ and $\omega= \pm 4 \pi, \pm 8 \pi \mathrm{rad} \mathrm{s}^{-1}$ are measurement noise corresponding to the rotation rate and its harmonics.

resultant flows are turbulent. They are highly irregular and contain energy in a wide range of scales (Supplementary Movie 1 for flow visualization).

A horizontal laser sheet illuminates a cross-section of the cylinder at a specific (variable) height. The laser sheet is swept vertically in the range $\Delta h=25.6 \mathrm{~cm}$ using a fast rotating mirror ('galvo'). Each vertical scan consists of 30 horizontal planes $(0.89 \mathrm{~cm}$ spacing) and lasts $46.7 \mathrm{~ms}$. A co-rotating camera captures the light scattered from the particles at $\sim 750 \mathrm{fps}$. The captured data is used for particle imaging velocimetry (PIV). The set-up provides $3 \mathrm{D}$ measurement of the horizontal velocity field, $\mathbf{u}_{\perp}$, inside a $25 \times 25 \times 26 \mathrm{~cm}^{3}$ volume at a $21.4 \mathrm{~Hz}$ rate (see Methods for more details).

We start by measuring the $2 \mathrm{D}$ flow field $(\Delta h=0 \mathrm{~cm})$ at different stages of turbulence build-up. The energy injection system is turned on at time $t=0$ while the fluid is at rest in the rotating frame (rigid body rotation). It was shown ${ }^{27}$ that at short times the $1 \mathrm{D}$ energy spectrum evolution is dominated by the arrival of the injected $(\sim 7 \mathrm{~cm})$ inertial waves at the measurement plane (Supplementary Information 2 and Supplementary Fig. 4a). At longer times (Fig. 2a and Supplementary Fig. 4b) the spectrum evolves towards its steady state via an inverse energy cascade (see ref. 5 for more details). The steady state spectrum is well fitted by $E_{k} \sim k^{-5 / 3}$. Both the spectrum evolution and its steady state are consistent with the wave turbulence predictions (see ref. 10 Ch. 3.2.4 and 9.2.3).

Next we measure the steady state turbulence and plot the average temporal Fourier transform of the energy, $E_{\omega}=1 / 2 T A \int\left(\left|\tilde{u}_{x}\right|^{2}+\right.$ $\left.\left|\tilde{u}_{y}\right|^{2}\right) \mathrm{d} x \mathrm{~d} y$, for various rotation rates. Here $\tilde{u}_{x}=\tilde{u}_{x}(x, y, \omega)$ and $\tilde{u}_{y}=\tilde{u}_{y}(x, y, \omega)$ are the temporal Fourier transforms of $u_{x}(x, y, t)$ and $u_{y}(x, y, t)$, respectively, $A$ is the measured area and $T$ is the total measurement time. In past works, this energy spectrum has received less attention than the spatial energy spectrum, $E_{k}$.
However, equation (1) provides information about the temporal variations in the flow which should be reflected in the temporal spectrum. The measured energy spectra have a characteristic shape (Fig. 2b): at low measured frequencies they have a well-defined power law $\omega^{-1.35 \pm 0.05}$, regardless of the rotation rate. The power law spans two decades, from the lowest measured frequency up to a cutoff frequency $\left(\omega^{\star}\right)$ (see Fig. $2 c$ for its definition). Above $\omega^{\star}$ the spectra have a sharp decrease in magnitude (inset in Fig. 2b). Plotting the cutoff frequency as a function of rotation rate (Fig. 2c) shows that $\omega^{\star} \approx 2 \Omega$, the frequency limit for inertial waves (see equation (1)). The relation holds for both the top and middle regions of the tank. These measurements indicate that the statistics of rotating turbulence is affected by the dispersion relation of inertial waves. However, such 2D measurements cannot tell if the statistics at $\omega<\omega^{\star}$, where most of the energy is located, is related to equation (1) in any way.

The next step is to directly measure the relation between $\omega$ and $\mathbf{k}$. As, according to equation (1), $\omega$ depends only on the angle of $\mathbf{k}$ with respect to the rotation axis $(\theta)$, a unique relation should exist only in the $(\omega, \theta)$ plane. Using the rapidly scanning laser sheet technique we measure the $3 \mathrm{D}$ horizontal velocity field $\mathbf{u}_{\perp}(x, y, z, t)$. The measured fields are Fourier transformed in three spatial coordinates and in time, leading to the fields $\tilde{\tilde{u}}_{x}(\mathbf{k}, \omega)$ and $\tilde{\tilde{u}}_{y}(\mathbf{k}, \omega)$, with the $4 \mathrm{D}$ energy spectrum defined as: $E(\mathbf{k}, \omega)=1 / 2\left(\left|\tilde{\tilde{u}}_{x}(\mathbf{k}, \omega)\right|^{2}+\left|\tilde{\tilde{u}}_{y}(\mathbf{k}, \omega)\right|^{2}\right)$. $E(\theta, \omega)$ is obtained by expressing $\mathbf{k}$ in spherical coordinates as $\mathbf{k}=(k, \theta, \varphi)$ and integrating $E(\mathbf{k}, \omega)$ over $\varphi$ and $k$ (the integration on $k$ is performed over a range in which the resolution in $\theta$ is sufficient-Supplementary Information 1 and Supplementary Fig. 1).

Figure 3 shows energy spectra that were obtained by integration over $\varphi$ and over four different ranges of $k$. The kinetic energy is localized along two well-defined curves in the $(\theta, \omega)$ plane. The 
a

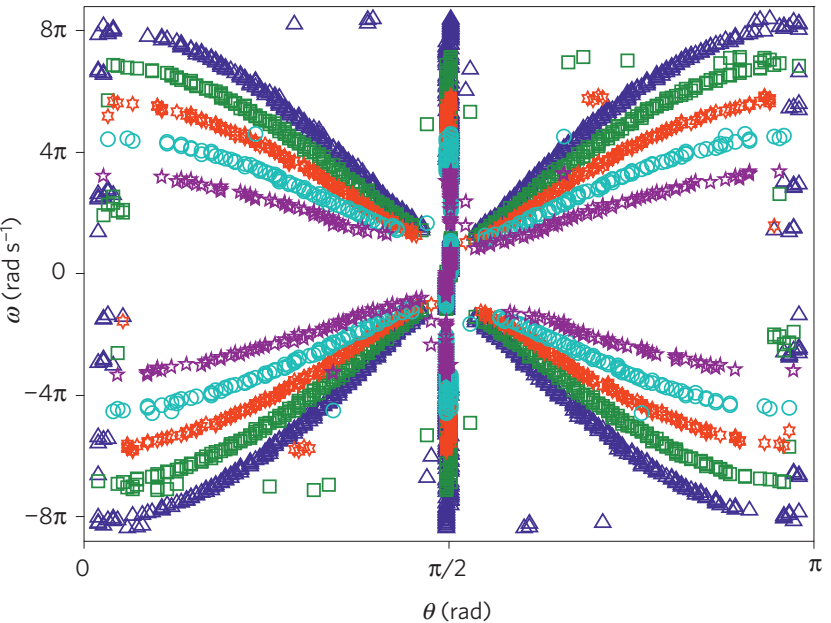

b

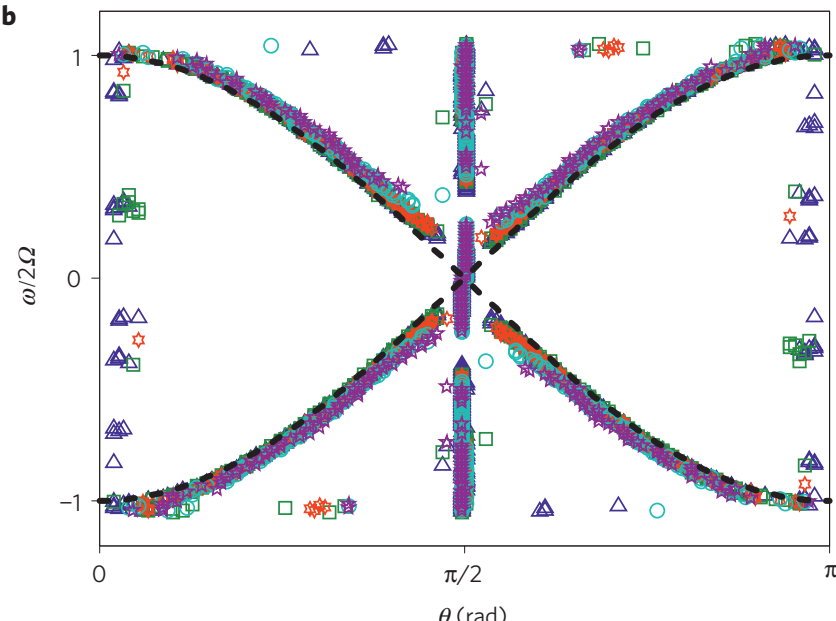

Figure 4 | Energy spectra peak location for various rotation rates. a, The location of energy peaks for all $\omega<2 \Omega$ for different rotation rates: $\Omega\left(\right.$ rad s ${ }^{-1}$ ) $=1.6 \pi$ (purple 5-pointed open star); $2.2 \pi$ (cyan open circle); $2.8 \pi$ (red 6-pointed open star); $3.4 \pi$ (green open square); and $4 \pi$ (blue open triangle). The peak values were extracted from data similar to that shown in Fig. 3, with integration across the full range of wavenumbers. $\mathbf{b}$, The same data as shown in $\mathbf{a}$, with the $\omega$ axis rescaled by $2 \Omega$. The black dashed lines are the expected rescaled dispersion relations: $\pm \cos (\theta)$. All experiments are for $h_{0}=6.85 \mathrm{~cm}$ and $\Delta h=25.9 \mathrm{~cm}$.

curves follow the dispersion relation (equation (1)) for $\omega<2 \Omega$, with practically no energy for $\omega>2 \Omega$, which is $8 \pi$ in this case. The similarity of the various subplots $(\mathrm{a}-\mathrm{d})$ indicates that, as expected, the shape of the curves is independent of $|k|$. The slight asymmetry in the power contained in the positive and negative branches of the dispersion relation (Fig. 3 c,d) is due to the net energy flux from the bottom of the tank to its top.

To further quantify the connection between the energy spectra of Fig. 3 and equation (1) we perform measurements of the full energy spectrum, $E(\theta, \omega)$, obtained by integration over the entire range $0.78<k<3.28 \mathrm{rad} \mathrm{cm}^{-1}$ (Supplementary Fig. 2). The spectra are obtained for different rotation rates, $\Omega$. Figure 4 a shows the maxima, per given frequency $\omega$, of the energy spectrum for various rotation rates. Rescaling $\omega$ by $2 \Omega$ (Fig. $4 \mathrm{~b}$ ) leads to an excellent data collapse and a very good agreement with the theoretical predictions given by equation (1) (black dashed lines). This is a clear and direct indication that the energy is contained in the form of linear inertial waves spanning almost all possible frequencies. This is true not just for the steady state, but also for evolving flows. Computing $E(\theta, \omega)$ during the spectrum build-up shows (Supplementary Information 3, Supplementary Fig. 5 and Movie 2) that the energy is contained in the form of inertial waves. Therefore, the energy transfer between scales, which is presented in Fig. 2a (and Supplementary Fig. 4b), is due to the interaction between inertial waves.

We have shown that steady deep rotating turbulence, of moderate Reynolds number, can be well described as a $3 \mathrm{D}$ wave turbulence. Linear inertial waves are the basic modes of the turbulent state. They transfer the injected energy and form a broad distribution of energy in both the length scale and timescale, while preserving their unique dispersion relation. Thus, the resultant turbulent state is characterized by a unique relation between spatial and temporal fluctuations of the flow. The entire 3D turbulent flow field, which was previously studied in an approximated 2D framework, is in fact an ensemble of interacting waves. The study of rotating turbulence can, therefore, be performed within a well-defined, fully $3 \mathrm{D}$ framework.

In the broader context, our work suggests the existence of wave turbulence in a 3D system - a system which is not naturally viewed as a wave system. As such, it provides crucial experimental support to this important theory.
The results presented here open the way for further quantitative studies. In particular, the pronounced temporal energy spectrum, which is close to $E_{\omega} \propto \omega^{-4 / 3}$, is not predicted by any theoretical work we are aware of. In fact, using the relation between $\omega$ and $\theta$ (equation (1), Fig. 4b) we can write $E(\cos (\theta)) \propto(\cos (\theta))^{-4 / 3}$. The singularity at $\theta=\pi / 2$ of this dependence is stronger than theoretical predictions ${ }^{14,15}$. The theoretical origin of this dependence and its implications should be studied theoretically and experimentally. Finally, questions regarding the range in parameter space in which rotating wave turbulence exists, and its relation to previously observed quasi $2 \mathrm{D}$ turbulence, call for further work.

\section{Methods}

We perform 2D PIV measurements on 30 horizontal planes using a rapidly scanning laser sheet. In each scan, the galvo starts at the smallest angle (laser sheet at lowest location: $\left.h_{0}-0.5 \Delta h\right)$ and scans upwards through the selected angles, triggered by the camera. After each frame the mirror rotates such that the laser sheet moves to the next height. After the laser sheet reaches the top layer $\left(h_{0}+0.5 \Delta h\right)$ the galvo rotates back to the lowest layer and starts over. The total rotation of the galvo is $0.8^{\circ}$ and the total optical distance to the tank is about $9 \mathrm{~m}$.

The PIV algorithm is self-written using MatLab, based on MatPIV and OpenPIV, and adjusted to work on a graphics processing unit (GPU). The PIV measurement is made between two pictures at the same height (2D velocity field). Our 2D measurements (Fig. 2) are made using a PIV window of $8 \times 8$ pixels with $50 \%$ overlap. For the spatial spectrum (Fig. 2 a) the measurements $(996 \times 996$ pixels) have a resolution of $0.23 \mathrm{~cm}$ with a $52 \mathrm{~cm}$ viewing field $(248 \times 248$ data grid size). In Supplementary Fig. 4 the resolution is $0.58 \mathrm{~cm}$ with a $43.2 \mathrm{~cm}$ viewing field $(600 \times 600$ pixels, window of $16 \times 16$ pixels, $74 \times 74$ data grid size). For the temporal spectrum (Fig. 2 b,c) the measurements $(470 \times 470$ pixels $)$ have a resolution of $0.23 \mathrm{~cm}$ with a $26.9 \mathrm{~cm}$ viewing field $(116 \times 116$ data grid size). The 3D measurements (Figs 3 and 4 ) are made with a window of $12 \times 12$ pixels, which results in a $0.32 \mathrm{~cm}$ resolution and a $24.3 \mathrm{~cm}$ viewing field. The $z$ axis has a $0.89 \mathrm{~cm}$ resolution with a total height of $25.9 \mathrm{~cm}$ (total data grid size: $77 \times 77 \times 30)$. Supplementary Fig. 3 has a resolution of $0.22 \mathrm{~cm}$ with a viewing field of $20.45 \mathrm{~cm}$ (window size of $10 \times 10$ with a $74 \times 74$ grid data size).

In the $3 \mathrm{D}$ measurements we perform PIV between every two sequential pictures at the same height. However, there is a difference in size between pictures at the top and bottom of the scanned volume (owing to the different distance between the camera and the laser sheet: from $0.66 \mathrm{~mm} / \mathrm{px}$ at the bottom layer to $0.53 \mathrm{~mm} / \mathrm{px}$ at the top layer). To use FFT we interpolate the PIV results according to the largest possible area which includes all heights (for example, the top layer size), and according to the bottom layer time.

Received 23 January 2014; accepted 8 May 2014; published online 8 June 2014 


\section{References}

1. Greenspan, H. P. The Theory of Rotating Fluids (Cambridge Univ. Press, 1968).

2. McEwan, A. D. Angular-momentum diffusion and initiation of cyclones. Nature 260, 126-128 (1976).

3. Hopfinger, E. J., Browand, F. K. \& Gagne, Y. Turbulence and waves in a rotating tank. J. Fluid Mech. 125, 505-534 (1982).

4. Baroud, C. N., Plapp, B. B., Swinney, H. L. \& She, Z. S. Scaling in three-dimensional and quasi-two-dimensional rotating turbulent flows. Phys. Fluids 15, 2091-2104 (2003).

5. Yarom, E., Vardi, Y. \& Sharon, E. Experimental quantification of inverse energy cascade in deep rotating turbulence. Phys. Fluids 25, 085105 (2013).

6. Chen, Q. N., Chen, S. Y., Eyink, G. L. \& Holm, D. D. Resonant interactions in rotating homogeneous three-dimensional turbulence. J. Fluid Mech. 542, 139-164 (2005).

7. Sen, A., Mininni, P. D., Rosenberg, D. \& Pouquet, A. Anisotropy and nonuniversality in scaling laws of the large-scale energy spectrum in rotating turbulence. Phys. Rev. E 86, 036319 (2012).

8. Zakharov, V. E., L'vov, V. S. \& Falkovich, G. Kolmogorov Spectra of Turbulence 1: Wave Turbulence (Springer, 1992).

9. Newell, A. C. \& Rumpf, B. in Annual Review of Fluid Mechanics, Vol. 43 (ed. Davis, S. H. M. P.) 59-78 (Annual Reviews, 2011).

10. Nazarenko, S. Wave Turbulence (Springer, 2011).

11. Smith, L. M. \& Waleffe, F. Transfer of energy to two-dimensional large scales in forced, rotating three-dimensional turbulence. Phys. Fluids 11, 1608-1622 (1999)

12. Cambon, C., Mansour, N. N. \& Godeferd, F. S. Energy transfer in rotating turbulence. J. Fluid Mech. 337, 303-332 (1997).

13. Cambon, C., Rubinstein, R. \& Godeferd, F. S. Advances in wave turbulence: Rapidly rotating flows. New J. Phys. 6, 73 (2004).

14. Bellet, F., Godeferd, F. S., Scott, J. F. \& Cambon, C. Wave turbulence in rapidly rotating flows. J. Fluid Mech. 562, 83-121 (2006).

15. Galtier, S. Weak inertial-wave turbulence theory. Phys. Rev. E 68 015301 (2003).

16. Pedlosky, J. Geophysical Fluid Dynamics (Springer, 1987).

17. Baroud, C. N., Plapp, B. B., She, Z. S. \& Swinney, H. L. Anomalous self-similarity in a turbulent rapidly rotating fluid. Phys. Rev. Lett. 88, 114501 (2002).

18. Ruppert-Felsot, J. E., Praud, O., Sharon, E. \& Swinney, H. L. Extraction of coherent structures in a rotating turbulent flow experiment. Phys. Rev. E 72, 016311 (2005).
19. Falcon, E. Laboratory experiments on wave turbulence. Discrete Contin. Dynam. Syst. Ser. B 13, 819-840 (2010)

20. Holt, R. G. \& Trinh, E. H. Faraday wave turbulence on a spherical liquid shell. Phys. Rev. Lett. 77, 1274-1277 (1996).

21. Berhanu, M. \& Falcon, E. Space-time-resolved capillary wave turbulence. Phys Rev. E 87, 033003 (2013).

22. Boudaoud, A., Cadot, O., Odille, B. T. \& Touzé, C. Observation of wave turbulence in vibrating plates. Phys. Rev. Lett. 100, 234504 (2008).

23. Mordant, N. Are there waves in elastic wave turbulence? Phys. Rev. Lett. 100, 234505 (2008).

24. Cobelli, P. et al. Space-time resolved wave turbulence in a vibrating plate. Phys. Rev. Lett. 103, 204301 (2009).

25. Bordes, G., Moisy, F., Dauxois, T. \& Cortet, P-P. Experimental evidence of a triadic resonance of plane inertial waves in a rotating fluid. Phys. Fluids 24, 014105 (2012)

26. Davidson, P. A., Staplehurst, P. J. \& Dalziel, S. B. On the evolution of eddies in a rapidly rotating system. J. Fluid Mech. 557, 135-144 (2006).

27. Kolvin, I., Cohen, K., Vardi, Y. \& Sharon, E. Energy transfer by inertial waves during the buildup of turbulence in a rotating system. Phys. Rev. Lett. 102, 014503 (2009).

28. Bewley, G. P., Lathrop, D. P., Maas, L. \& Sreenivasan, K. R. Inertial waves in rotating grid turbulence. Phys. Fluids 19, 071701 (2007)

\section{Acknowledgements}

This work was supported by the Israel Science Foundation, Grant No. 81/12.

\section{Author contributions}

E.Y. designed, measured and analysed the data, E.S. initiated the experiment. Both authors wrote the paper.

\section{Additional information}

Supplementary information is available in the online version of the paper. Reprints and permissions information is available online at www.nature.com/reprints.

Correspondence and requests for materials should be addressed to E.S.

\section{Competing financial interests}

The authors declare no competing financial interests. 